\title{
Contribution from EM Surveys to a Regional Groundwater Resource Quantification Project in an Hard Rock Area.
}

\author{
Roy $\mathrm{J}^{1}$., Lubczynski M.W' $\mathrm{W}^{2}$, Sporry R.J $\mathrm{J}^{3}$., Hugens W.A $\mathbf{A}^{4}$. \\ 1,3.4: ITC, 2628EB Delft, The Netherlands, 2: ITC, 7500AA Enschede, The Netherlands
}

\section{INTRODUCTION}

This note is dealing with two key concepts: the quantification of groundwater (GW) resources on one hand and the use of electromagnetic (EM) techniques on the other; these are the objects of this introduction.

Groundwater resource quantification. - Regional GW resource quantification, considering the heterogeneous and anisotropic distribution of the subsurface hydraulic properties, is best performed with the use of numerical models. In the case of hard rock terrains, the heterogeneity of the formations from a hydraulic point of view, is of particular significance so that the interpolation and/or extrapolation of hydraulic parameters over a selected area from scant empirical data points can not be made reliable in a simple and direct way. There are various strategies used to overcome this problem. One of them is the equivalent porous medium (EPM) scheme where at a sufficiently large scale, the hydraulic properties of an heterogeneously fractured set of formations is replaced by a porous medium having similar hydraulic properties. Therefore, scale problems are prominent when dealing with the transmissivity. At large cartographic scales, the model elements can have near zero transmissivity if solid granite blocks are encountered, but adjoining elements can have high transmissivity in case of deep fractures and associated weathering. Therefore, very often, in the modelling procedure a distinct increase of effective transmissivity with increasing scale of observation is reported (Sanchez Vila et al, 1996). Thus, the EPM models give a poor representation of the reality if applied in a routine manner, in which the transmissivity is regarded as an independent variable and recharge is treated as a calibrated model parameter (dependent variable). To solve the problem of getting representative values for the hydraulic conductivity/transmissivity the estimation problem is attacked from the back end (Lubczynski, 1997): the recharge is evaluated, and then the hydraulic parameters are fitted to the recharge data set. The spatial distribution of the recharge values themselves is evaluated using a model based on Remote Sensing (RS) data and Geographic Information System (GIS) techniques. In this model the distribution of various terrain's characteristics is determined from RS data while their influence on the recharge potential is modelled and used in the GIS environment to produce a relative recharge map which first is validated (quantified) by the recharge well hydrographs and then used in the design of the numerical $\mathrm{GW}$ model. The actual distributions of hydraulic properties are adjusted during a calibration procedure taking into account field data related to observed head distribution and other available information such as field determined hydraulic properties, topographic and hydrographic data and also information derived from geophysical surveys' results. This scheme of regional GW resources quantification is currently being applied to a watershed in the Salamanca area, Spain (Rio Tormes watershed) where the fresh basement is made of granite.

Electromagnetic surveys. - Electromagnetic surveys, on the other hand, as used for GW 
quantification projects have often been made using configurations with loops of finite size (i.e. not equivalent to a dipole at the scale of the problem) normally with time domain electromagnetic systems (TDEM) for the determination of the geoelectrical stratigraphy, in salt water/fresh water interface problems etc. There are however practical limitations in the use of these configurations at shallow depths and for high lateral resolution work. EM systems using dipolar sources have been used for this last category of tasks and these EM systems are normally operated in the frequency domain. One well-known implementation of an EM system designed for high resolution shallow work is the EM conductivity meter (McNeil, 1980). Recent detailed surveys using this type of instrument are reported for the mapping of near surface conductivity changes with high spatial resolution (Sporry et. al., 1995, 1997). EM conductivity meter data provide information on the lateral changes in conductivity but their sounding (i.e. vertical distribution of conductivities) capability is limited. The work reported here used a multifrequency EM horizontal coplanar loops system (Slingram) with and without a rigid frame to map the subsurface resistivity in a soundingprofiling mode. Under favourable circumstances such survey supplies information on the localisation and dimensions of the main formations with significant hydraulic conductivity.

\section{METHODOLOGY}

Relative size of intercoil spacing and skin depth. - In the project reported here, the Slingram EM system was operated with coil spacings sometimes smaller and other times larger than the skin depth according to the frequency used and the ground conductivity. This puts tighter constraints at the data inversion step than when the coil spacing is always much smaller than the skin depth.

Sounding/profiling. - The EM data was acquired along profiles of characteristic interest to the groundwater quantification aspect of the project. It is considered here as a sounding/profiling method because the multifrequency/multiseparation data allows some determination of the vertical distribution of conductivity below the ground surface while at the same time, because the data was acquired every 60 meters along the profile, lateral changes are detectable.

Rigid frame. - For operation of the Slingram EM set with short coil spacing, a rigid frame was used to minimise the difficulties of maintaining a constant intercoil coupling (distance and orientation) in the coplanar configuration; a similar solution was reported earlier by Westerberg (1965) but with the current technology a much wider range of frequency is available in commercial EM systems: here from $444 \mathrm{hz}$ to $56 \mathrm{khz}$ (Model MAX-MIN I8S with the MMC digital data recorder from Apex Parametrics Ltd.). Figure 1 illustrates the setup used for the current project: the flat coil up front is the transmitter coil while the receiver and its associated digital data acquisition system is at the far end. Because of the rigidity of the frame, the transmitter-receiver coupling is nearly constant. The need for a stabilisation scheme at short Slingram intercoil spacing is illustrated as follows: at a spacing of 5 metres, a distance error of only $5 \mathrm{~cm}$ produces a $3 \%$ error while over a .44 to $56 \mathrm{khz}$ frequency interval, a $100 \Omega \mathrm{m}$ homogeneous ground will have a response smaller than $2 \%$.

Phase-gain calibration. - An interesting feature of the Slingram model used in this project is the availability of a gain and phase calibration facility. Essentially it allows to alter the magnitude of the in-phase compensation signal by a known percentage during the calibration step and thus to evaluate the actual phase and gain errors under survey conditions. This information is digitally recorded and used at the data processing step to correct numerically the data set for any residual hardware miscalibration of the system.

Real component correction. - The EM data were acquired at two intercoil spacings: 5 and 120 metres. In the second case, over flat areas, the intercoil distance and orientation was 
maintained over flat areas by using a measured cable for distance evaluation and using the instrument's levels to maintain the coils horizontal. This, however, produced a systematic measurement noise on the real component which was reduced using the following scheme: .1 for simple layered cases with an horizontal coplanar loops Slingram, assuming small to moderate coil spacing and layers' conductivity, and for the lowest frequencies, the real component of the response is smaller than the quadrature component and in most cases is nearly equal to zero; .2 at a given survey point the coupling error will be approximately the same at all the frequencies used (this assumes that most of the error is due to the initial positioning rather than changes in position while the measurements are in progress); .3 therefore, at all survey points, the value of the real component corresponding to the lowest frequency $(444 \mathrm{hz}$ ) or the average from the two lowest frequencies (when the quadrature response at $444 \mathrm{hz}$ was negligible) was subtracted from the real component of the readings at all frequencies. This reduced significantly the in-phase noise in the data set and allowed data inversion; without this correction very large misfits were observed whenever data inversion was attempted.

Data inversion. - As a final step the corrected data was written to a file in a format compatible with the data inversion tool used (Anderson, 1992); the EM data inversion is done in an interactive mode using a PC and Anderson's INVDP numerical data modelling program.

\section{RESULTS}

Data interpretation. - The EM sounding/profiling responses could be sorted in the following quite distinct cases: .1 conductive one or two layers case; .2 intermediate conductivity one to three layers; .3 no significant EM response: in this case, the subsurface formation is too resistive to be measured by this EM technique; if quantitative information is required for this last case, another technique, such as galvanic resistivity survey, is suggested. In the first case, one or two conductive layer(s) are observed and the conductivity corresponds to clay rich sediments accumulated in a structural depression along the valley axis. In the second case a model with one, two or three layers is fitted to the data set, sometimes with a higher resistivity top layer and one or two layers of moderate resistivities assumed to be due to weathered or fractured granite below the water table. Finally in the third case, the response is outside the measuring range of the system; the presence of high resistivity materials is assumed to be due to unfractured granite. The dimensions of the individual layers controlling these three type of responses are currently being determined. Figure 2 is an example of data inversion for a reading point near the Western limit of the survey profile. In this case a two layers model was fitted to the data with a 38 meters thick first layer $(840 \Omega \mathrm{m})$ and a $315 \Omega \mathrm{m}$ second layer. Finally the Figure 3 shows a coarse cross-section along one of the profile. On the Western side one could observe the layering due to the fractured hard rock, in the centre, the structural depression filled with clayey sediments is noticeable; this is the area where the largest EM responses were observed. Finally the Eastern extremity is not quantitatively mapped; it is estimated that the resistivity of the formation is higher than $\sim 700 \Omega \mathrm{m}$ everywhere in this last sector and that it is due to unfractured hard rock. Finaly, on this profile the $5 \mathrm{~m}$ Slingram data was mostly useful to map the low resistivity depression and the position of the fresh rock to the East while the $120 \mathrm{~m}$ data was used for the intermediate resistivities West of the depression.

Methodology. - This multifrequency/multiseparation EM sounding/profiling technique allows a rapid determination of the subsurface geoelectric section although a streamlined data path 
from acquisition to integration of results in a groundwater quantification project is still being developed. This test has permitted to detect a number of areas where improvements were needed in particular in finding out a lighter way of design for a rigid structure, a more dependable procedure for the data acquisition specially at the high frequency end and the need for an improved implementation of the data correction, interpretation and display and integration procedures. This is an ongoing project and such improvements are being added during the project lifetime. A scale factor was reported at the outset, from the hydraulic point of view; a scale effect is also observed from the EM point of view when analysing Slingram data sets acquired at different coil spacing. At $120 \mathrm{~m}$ spacing as is noticed on Figure 2 there is a misfit with a $1 \mathrm{D}$ layered model probably because of the size of the heterogeneities. It has to be pointed out that during the numerical model calibration, apart from the independent variable type of data like hydraulic head and recharge distribution, other hydrogeological data can be used interactively to improve the accuracy and reliability of the model. These data include mainly hydrostratigraphy and media properties for which the Slingram EM can contribute efficiently.

\section{CONCLUSION}

While improvements are still required on the procedures, this class of EM surveys may contribute significantly to the detection, localisation and evaluation of the dimensions of the various formations having a significant hydraulic conductivity: in hard rock areas, these are usually detectable by their contrast in electric conductivity. This information will be of direct use in the hydrogeological modelling of the area.

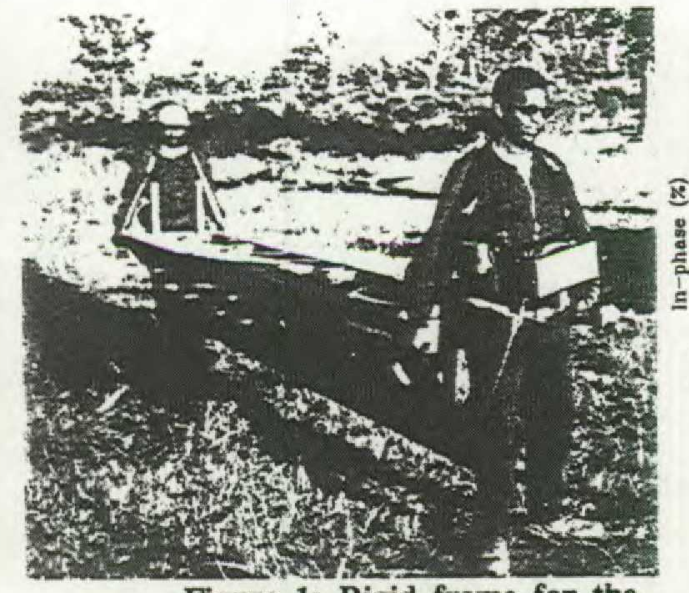

Figure 1: Rigid frame for the Slingram EM set at an intercoil spacing of 5 meters.

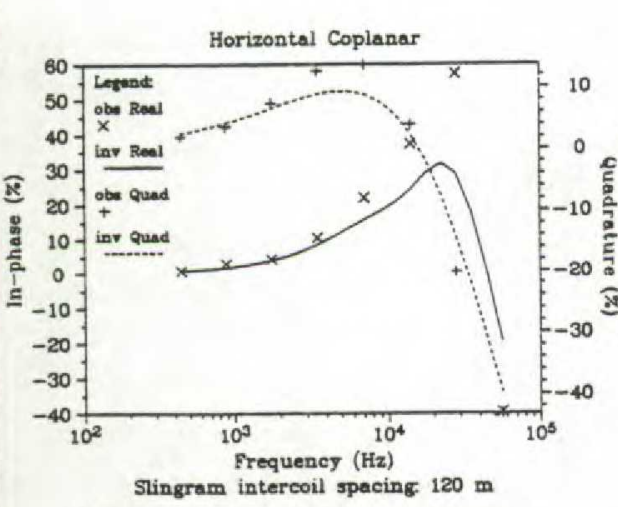

Figure 2: Exampie of the inversion of an EM data point

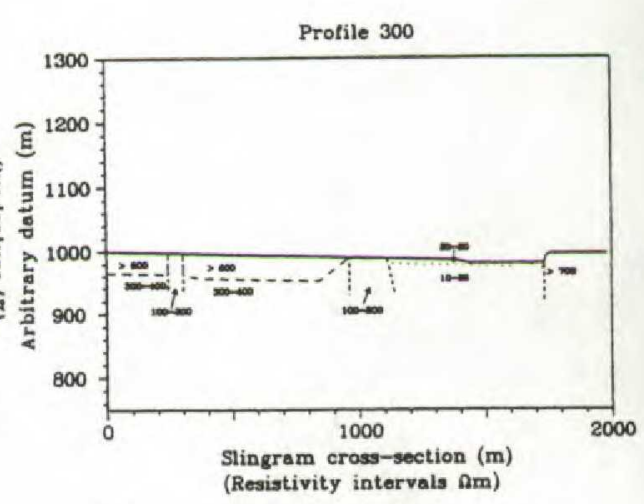

Figure 3: Cross section along a surveyed profile

Anderson W.L., 1992, Interactive inversion of dipole loop-loop electromagnetic data for layered earth models using numerical integration and complex image theory, USGS OFR-92-553.

Lubczynski M.W., 1997, Application of numerical flow modelling combined with remote sensing and GIS techniques for the quantification of regional groundwater resources in hard rock terrain; [Hard rock hydrosystems, Proceedings of Rabat Symposium, S-2, May 1997]. IAHS Publ. MO.241,1997

McNeil J.D., 1980, Electromagnetic terrain conductivity measurement at low induction numbers, TN-6, Geonics Ltd.

Sanchez-Vila, X., Carrera, J. and Girardi, J.P. (1996) Scale effects in transmissivity. J. Hydrol. 183, 1-22.

Sporry R.J., Roy, J., Hugens W.A. and Sibhat N., High resolution geophysical sensing and imaging, EEGS 1st European meeting, Torino, 1995

Sporry R.J., Marques da Costa A., Desta G., Roy, J., and Hugens W.A., A high density sampling survey of shallow karst features using EM and magnetic profiling techniques, EEGS $3^{\text {rd }}$ European Meeting, Aarhus, 1997.

Westerberg K., 1965, The 'Beam-Slingram': a new portable EM instrument for ore prospecting; Geoexploration 3 , $149-154$. 